Exploring Stochastic Laws. pp. 129-134.

Eds. A.V. Skorokhod and Yu.V. Borovskikh

(C) VSP Utrecht, 1995

\title{
Bounds for absolute moments of order statistics
}

\author{
NADEZHDA V. GRIBKOVA \\ Department of Applied Mathematics, Transport University, \\ 199031, St.Petersburg, Moskovsky Prospect 9, Russia
}

Received February 23, 1995

\begin{abstract}
The bounds for absolute moments of order statistics are established. Let $X_{1}, \ldots, X_{n}$ be independent identically distributed real-valued random variables and let $X_{1: n} \leq \cdots \leq X_{n: n}$ be the corresponding order statistics. The absolute moments $\mathbf{E}\left|X_{i: n}\right|^{k}, k>0$, are estimated via the absolute moment $\mathbf{E}\left|X_{1}\right|^{\delta}, \delta>0$, for all $i$ such that $k \delta^{-1} \leq i \leq n-k \delta^{-1}+1$ with order $\left(n^{2} i^{-1}(n-i)^{-1}\right)^{k \delta^{-1}}$ in $i$ and $n$. These estimates are able to be of some use as a tool to argue in different probability limit theorems.
\end{abstract}

Keywords: order statistics; absolute moments; asymptotic bounds.

MSC: 62G30, 60E15.

Let $X_{1}, \ldots, X_{n}$ be independent identically distributed real-valued random variables with common distribution function $F$, and let $X_{1: n} \leq \cdots \leq X_{n: n}$ denote the corresponding order statistics. Let $k$ and $\delta$ be arbitrary positive numbers. Put $\rho=k \delta^{-1}$ and set $g(u)=u(1-u)$.

Theorem 1. For all $n \geq 2 \rho+1$ and for all $i$ such that $\rho \leq i \leq n-\rho+1$ the following inequality holds

$$
\mathbf{E}\left|X_{i: n}\right|^{k}<C(\rho)\left\{\mathbf{E}\left|X_{1}\right|^{\delta} g^{-1}\left(\frac{i}{n+1}\right)\right\}^{\rho}
$$

where one can put $C(\rho)=2 \sqrt{\rho} \exp (\rho+7 / 6)$.

Consequence. For arbitrary $0<\alpha<\beta<1$ and for all $n$ and $i$ such that $\rho \leq n \alpha<i<n \beta \leq n-\rho+1$, the following inequality holds

$$
\mathbf{E}\left|X_{i: n}\right|^{k}<C(\alpha, \beta, \rho)\left\{\mathbf{E}\left|X_{1}\right|^{\delta}\right\}^{\rho},
$$

where $C(\alpha, \beta, \rho)$ is constant, and depends only on $\alpha, \beta$ and $\rho$. 
Estimate (11) was applied in Gribkova (1989, 1994) for obtaining estimates in the Central Limit Theorem for $L$-statistics, and it was applied in Korolyuk and Borovskikh (1993) for the analysis of the asymptotic behavior of random permanent measures for $L$-statistics. It should be noted that many inequalities and other auxiliary results for order statistics are included in Helmers (1982), van Zwet (1970), Shorack and Wellner (1986).

Proof of Theorem 1. To begin with let us note that by Chebyshev's inequality,

$$
\left(F^{-1}(u)\right)^{\delta}(1-u) \leq \mathbf{E}\left|X_{1}\right|^{\delta}
$$

for $u \geq F(0)$ and

$$
\left|F^{-1}(u)\right|^{\delta} u \leq \mathbf{E}\left|X_{1}\right|^{\delta}
$$

for $u<F(0)$ where

$$
F^{-1}(u)=\inf \{x: F(x) \geq u\} .
$$

We first assume that $\rho+1 \leq i \leq n-\rho$. Then we obtain

$$
\begin{aligned}
\mathbf{E}\left|X_{i: n}\right|^{k}= & \frac{1}{B(i, n-i+1)} \int_{0}^{1}\left|F^{-1}(u)\right|^{k} u^{i-1}(1-u)^{n-i} d u \\
\leq & \frac{\left\{\mathbf{E}\left|X_{1}\right|^{\delta}\right\}^{\rho}}{B(i, n-i+1)}\left(\int_{0}^{F(0)} u^{i-\rho-1}(1-u)^{n-i} d u\right. \\
& \left.+\int_{F(0)}^{1} u^{i-1}(1-u)^{n-\rho-i} d u\right) \\
< & \left\{\mathbf{E}\left|X_{1}\right|^{\delta}\right\}^{\rho}\left(\frac{B(i-\rho, n-i+1)}{B(i, n-i+1)}+\frac{B(i, n-\rho-i+1)}{B(i, n-i+1)}\right) .
\end{aligned}
$$

To evaluate the ratios of beta functions on the right hand side of (2), we apply the inequalities

$$
\sqrt{2 \pi} x^{x+1 / 2} e^{-x}<\Gamma(1+x)<\sqrt{2 \pi} x^{x+1 / 2} e^{-x+1 /(12 x)},
$$

where $x \in \mathbb{R}, x>0$, which follows from Stirling's expansion. By using (3), we will prove that for all $\rho>0$ and for all $i \geq \rho+1$

$$
B(i-\rho, n-i+1) / B(i, n-i+1)<e^{1+7 / 6}(n / i)^{\rho} .
$$

Relation (4) and the symmetry imply that

$$
B(i, n-\rho-i+1) / B(i, n-i+1)<e^{1+7 / 6}(n /(n-i+1))^{\rho}
$$

for all $\rho>0$ and for all $i \leq n-\rho$. Together (2) and bounds (44)-(51) yield (1) in the case $\rho+1 \leq i \leq n-\rho$. Now we will prove (4). We have

$$
B(i-\rho, n-i+1) / B(i, n-i+1)=\frac{\Gamma(i-\rho) \cdot \Gamma(n+1)}{\Gamma(i) \cdot \Gamma(n-\rho+1)} .
$$


First suppose that $i \geq \rho+2$, so therefore $i-\rho-1 \geq 1$, and applying (3i) on the right hand side of (6), we find that (6) is less than

$$
e^{1 / 6}\left(\frac{(i-\rho-1) n}{(n-\rho)(i-1)}\right)^{1 / 2}\left(\frac{n}{i-1}\right)^{\rho}\left(\frac{n}{n-\rho}\right)^{n-\rho}\left(\frac{i-\rho-1}{i-1}\right)^{i-\rho-1} .
$$

To continue let us note that

$$
\begin{gathered}
\left(\frac{n}{n-\rho}\right)^{n-\rho}<e^{\rho}, \\
\left(\frac{(i-\rho-1) n}{(n-\rho)(i-1)}\right)^{1 / 2}<1, \quad\left(\frac{i-\rho-1}{i-1}\right)^{i-\rho-1}<e^{-\rho+\rho^{2} /(i-1)},
\end{gathered}
$$

so the magnitude (77) is less than

$$
e^{1 / 6+\rho^{2} /(i-1)}\left(\frac{n}{i-1}\right)^{\rho}<e^{\rho+7 / 6}\left(\frac{n}{i}\right)^{\rho} .
$$

Now we will estimate (6) for the case

$$
\rho+1 \leq i \leq \rho+2 .
$$

Then we have $1 \leq i-\rho<2, \Gamma(i-\rho) \leq 1$, and so the right hand side of (6) is not larger than

$$
\Gamma(n+1) /(\Gamma(i) \Gamma(n-\rho+1)) .
$$

If $\rho$ is an integer, then $\Gamma(n+1) / \Gamma(n-\rho+1)<n^{\rho}$ and $i$ from (9) is $i=\rho+1$. For such $i$ we have

$$
\Gamma(n+1) /(\Gamma(i) \Gamma(n-\rho+1))<\frac{n^{\rho} e^{\rho}}{\sqrt{\rho} \rho^{\rho}}<e^{\rho+1}(n / i)^{\rho},
$$

and so (4) follows. And further, if $\rho$ is not an integer, then $i$ from (9) is $i=[\rho]+2$ (here and further $[k]$ means the whole part of positive $k$ ). Suppose at first that $[\rho]=0$. Then $i=2$ and (10) is equal to

$$
\Gamma(n+1) / \Gamma(n-\rho+1))<e^{1 / 12} n^{\rho} \sqrt{1+\rho}<e^{1 / 12+\rho / 2+1}(n / i)^{\rho},
$$

and so (4) is true. Now we will suppose that $|\rho| \geq 1$, then we have

$$
\begin{aligned}
\Gamma(n+1) /(\Gamma(i) & \cdot \Gamma(n-\rho+1))<e^{1 / 12} n^{\rho} \sqrt{1+\rho} \Gamma^{-1}([\rho]+2) \\
& <e^{1 / 12} \sqrt{\frac{1+\rho}{1+[\rho]}} \frac{1}{\sqrt{2 \pi}}\left(\frac{n}{1+[\rho]}\right)^{\rho} e^{\rho}\left(\frac{e}{1+[\rho]}\right)^{1+[\rho]-\rho} \\
& <\frac{1}{\sqrt{2 \pi}}\left(\frac{3}{2}\right)^{3 / 2} e^{\rho+1 / 12}\left(\frac{n}{1+[\rho]}\right)^{\rho} \\
& <e^{\rho+1}(n / i)^{\rho} .
\end{aligned}
$$


Together bounds (6) $-(8)$ and (11) $-(13)$ imply (44). Thus we have proved our theorem for the case $\rho+1 \leq i \leq n-\rho$ for all $\rho>0$. It still remains to establish (1) for the extreme values of $i$. Now let

$$
\rho \leq i<\rho+1 .
$$

If $\rho \leq 1$ (i.e. $k \leq \delta$ ), then $i$ from (14) is $i=1$. Now by Hölder's inequality we obtain

$$
\begin{aligned}
\mathbf{E}\left|X_{1: n}\right|^{k} & =B^{-1}(1, n) \int_{-\infty}^{\infty}|x|^{k}(1-F(x))^{n-1} d F(x) \\
& \leq B^{-1}(1, n)\left\{\int_{-\infty}^{\infty}|x|^{\delta} d F(x)\right\}^{\rho} \\
& =n^{\rho}\left\{\mathbf{E}\left|X_{1}\right|^{\delta}\right\}^{\rho},
\end{aligned}
$$

and so (1) is true. Now let $\rho>1$ (i.e. $k>\delta$ ). In $\rho$ is an integer, then $i$ from (14) is $i=\rho$. Now for that $i$ we have

$$
\begin{aligned}
\mathbf{E}\left|X_{1: n}\right|^{k} & =B^{-1}(\rho, n-\rho+1) \int_{-\infty}^{\infty}|x|^{k} F^{\rho-1}(x)(1-F(x))^{n-\rho} d F(x) \\
& \leq B^{-1}(\rho, n-\rho+1)\left\{\mathbf{E}\left|X_{1}\right|^{\delta}\right\}^{\rho-1} \int_{-\infty}^{\infty}|x|^{\delta}(1-F(x))^{n-2 \rho+1} d F(x) \\
& \leq B^{-1}(\rho, n-\rho+1)\left\{\mathbf{E}\left|X_{1}\right|^{\delta}\right\}^{\rho} .
\end{aligned}
$$

Further,

$$
\begin{aligned}
B^{-1}(\rho, n-\rho+1) & =\Gamma(n+1) /(\Gamma(\rho) \cdot \Gamma(n-\rho+1)) \\
& <e^{1 / 12-1} \frac{1}{\sqrt{2 \pi}} \sqrt{\rho-1} \frac{n^{n}}{(\rho-1)^{\rho}(n-\rho)^{n-\rho}} \sqrt{\frac{n}{n-\rho}} \\
& <e^{1 / 12-1} \frac{\sqrt{\rho-1}}{\sqrt{2 \pi}}\left(\frac{n}{\rho-1}\right)^{\rho} e^{\rho} \sqrt{2} \\
& <\frac{1}{\sqrt{\pi}} e^{1 / 12+\rho+1} \sqrt{\rho-1}(n / i)^{\rho} .
\end{aligned}
$$

Now we assume that $\rho>1$ is not an integer. Then $i$ from (14) is $i=[\rho]+1$. For such $i$ we obtain

$$
\begin{aligned}
\mathbf{E}\left|X_{1: n}\right|^{k} & =B^{-1}([\rho]+1, n-[\rho]) \int_{-\infty}^{\infty}|x|^{k} F^{[\rho]}(x)(1-F(x))^{n-[\rho]-1} d F(x) \\
& \leq \frac{\left\{\mathbf{E}\left|X_{1}\right|^{\delta}\right\}^{[\rho]}}{B([\rho]+1, n-[\rho])} \int_{-\infty}^{\infty}|x|^{\delta(\rho-[\rho])}(1-F(x))^{n-2[\rho]-1} d F(x) .
\end{aligned}
$$


By Hölder's inequality the right hand side of (17) does not exceed

$$
\begin{aligned}
& \frac{\left\{\mathbf{E}\left|X_{1}\right|^{\delta}\right\}^{[\rho]}}{B([\rho]+1, n-[\rho])} \cdot \frac{\left\{\mathbf{E}\left|X_{1}\right|^{\delta}\right\}^{\rho-[\rho]}}{\{B(1, n-2[\rho])\}^{\rho-[\rho]-1}} \\
= & \left\{\mathbf{E}\left|X_{1}\right|^{\delta}\right\}^{\rho} \frac{n !(n-2[\rho])^{\rho-[\rho]-1}}{[\rho] !(n-[\rho]-1) !} \\
\leq & \left\{\mathbf{E}\left|X_{1}\right|^{\delta}\right\}^{\rho} \frac{n^{\rho}}{[\rho] !}\left(\frac{n}{n-2[\rho]}\right)^{[\rho]+1-\rho} .
\end{aligned}
$$

If $[\rho]=1$, then the right hand side of (18) is equal to

$$
\left\{\mathbf{E}\left|X_{1}\right|^{\delta}\right\}^{\rho} n^{\rho}\left(1+\frac{2}{n-2}\right)^{2-\rho}
$$

Since $n-2 \geq 2$ (because $n \geq 2 \rho+1>3$ ), the latter quantity is less than

$$
\left\{\mathbf{E}\left|X_{1}\right|^{\delta}\right\}^{\rho} n^{\rho} \cdot 2 .
$$

Thus for $i=[\rho]+1=2$ we have the inequality

$$
\left\{\mathbf{E}\left|X_{1}\right|^{\delta}\right\}^{\rho} n^{\rho} \cdot 2<2 e^{\rho}\left\{\frac{n^{2}}{2(n-2)}\right\}^{\rho},
$$

which implies (11). It remains to consider the case $[\rho] \geq 2$. In view of (3) the right hand side of (18) is less than

$$
\left\{\mathbf{E}\left|X_{1}\right|^{\delta}\right\}^{\rho} \frac{1}{\sqrt{2 \pi}} e^{[\rho]}\left(\frac{n}{[\rho]}\right)^{\rho} \sqrt{[\rho]}\left(2+\frac{1}{[\rho]}\right)^{[\rho]+1-\rho}<\left\{\mathbf{E}\left|X_{1}\right|^{\delta}\right\}^{\rho} \sqrt{[\rho]}(n /[\rho])^{\rho} e^{[\rho]} .
$$

So for $i=[\rho]+1$ we get the estimate

$$
\begin{aligned}
\mathbf{E}\left|X_{i: n}\right|^{k} & <\left\{\mathbf{E}\left|X_{1}\right|^{\delta}\right\}^{\rho} e^{\rho} \sqrt{\rho} e^{\frac{\rho-[\rho]}{[\rho]}-(\rho-[\rho])+1}\left(\frac{n}{i}\right)^{\rho} \\
& <e^{\rho+1} \sqrt{\rho}\left\{\mathbf{E}\left|X_{1}\right|^{\delta}\right\}^{\rho}\left(g^{-1}\left(\frac{i}{n+1}\right)\right)^{\rho} .
\end{aligned}
$$

Thus, inequality (1) is proved for all $i$ such that $\rho \leq i<\rho+1$ and all $\rho>0$, and in a standard way consisting in the change of the sign of the original random variables, we obtain that (11) is also valid for all $i$ such that $n-\rho<i \leq n-\rho+1$ and all $\rho>0$. The theorem is proved.

\section{References}

Gribkova, N. V. (1989). On the rate of convergence to the normal law of finitely truncated linear combinations of order statistics. Theory Probab. Appl., 33(4):726-729. 
Gribkova, N. V. (1994). On analogues of Berry - Esseen inequality for truncated linear combinations of order statistics. Theory Probab. Appl., 38(1):142-149.

Helmers, R. (1982). Edgeworth expansions for linear combinations of order statistics. Mathmatical Centre Tracts, 105, CWI, Amsterdam.

Korolyuk, V. S. and Borovskikh, Yu. V. (1993). Random permanents. Inst. of Mathem., Kiev (in Russian).

Shorack, G. R. and Wellner, J. A. (1986). Empirical processes with application in statistics. Wiley, New York.

van Zwet, W. R. (1970). Convex transformations of random variables, 2-nd ed. Mathmatical Centre Tracts, 7, CWI, Amsterdam. 